\title{
Stress concentration and optimal design of pinned connections
}

\author{
Pedersen, Niels Leergaard
}

Published in:

The Journal of Strain Analysis for Engineering Design

Link to article, DOI:

$10.1177 / 0309324719842766$

Publication date:

2019

Document Version

Peer reviewed version

Link back to DTU Orbit

Citation (APA):

Pedersen, N. L. (2019). Stress concentration and optimal design of pinned connections. The Journal of Strain Analysis for Engineering Design, 54(2), 95-104. https://doi.org/10.1177/0309324719842766

\section{General rights}

Copyright and moral rights for the publications made accessible in the public portal are retained by the authors and/or other copyright owners and it is a condition of accessing publications that users recognise and abide by the legal requirements associated with these rights.

- Users may download and print one copy of any publication from the public portal for the purpose of private study or research.

- You may not further distribute the material or use it for any profit-making activity or commercial gain

- You may freely distribute the URL identifying the publication in the public portal

If you believe that this document breaches copyright please contact us providing details, and we will remove access to the work immediately and investigate your claim 


\title{
Stress concentration and optimal design of pinned connections
}

\author{
Niels Leergaard Pedersen \\ Dept. of Mechanical Engineering, Solid Mechanics \\ Technical University of Denmark \\ Nils Koppels Allé, Building 404, DK-2800 Kgs. Lyngby, Denmark \\ email: nlp@mek.dtu.dk
}

\begin{abstract}
A pinned connection or lug joint is a common connection type used both in civil engineering and mechanical engineering. In civil engineering this connection is i.e. used for assembling truss members and in mechanical engineering this connection type is widely used in machine elements. The standard design is with a circular pin. The stress concentration factor size depends on the tolerances between pin and assembled parts and also by the 3D design. Relatively different maximum stress values are seen depending on the modelling being done in 2D (with assumptions) or in full 3D. The focus in the present paper is on the 2D design and minimizing the maximum stress. It is shown that not only the contact geometry is important for reducing the stress, the external design is equally important. By finite element analysis including contact modelling it is in the present paper shown that reductions in the stress concentration factor of up to $18 \%$ are possible.
\end{abstract}

Key words: Machine elements, pinned connection, lug joint, stress concentration, FEA, 2D.

\section{Introduction}

n many practical applications the assembly of different parts is done by pinned connections which are also termed lug joints. The simple assembly makes this type of connection preferable in cases where quick assembly and disassembly is needed, e.g. for the handling of shipping containers. In many civil engineering applications the lug joint is permanent, e.g. in truss assemblies ensuring that only axial load is transferred to the truss. Also in electrical connections this type of connection is used but the present paper focus is related to applications where it is the mechanical strength that is important. The loading of the connection is assumed to be cyclic so that the strength is related to fatigue and therefore to the maximum stress in the connection. In machine elements the pinned connections are typically used for shaft hub connections ensuring that the power can be transferred between them, in many cases the pin can also serve as a safety component if it is made by a significantly weaker material than the hub and shaft. In all of the mentioned cases the pinned connection is a subset of positive connections. The pin design is for machine elements generally controlled by standards, see e.g. DS/EN ISO 2338 (1998).

The design of pinned connections is essentially 3 dimensional although most focus is on the cross sectional design that is typically extruded into a given thickness. The cross sectional 
design is circular and stress concentration factor charts for tight fits (no clearance) can be found in e.g. Pilkey (1997). The typical used modelling assumption is that it is sufficient to use 2D with a further assumption of either plane stress (if the contact length in axial direction is relatively small) or plane strain (if the thickness is relatively large). For practical cases where the connection thickness is comparable to the pin diameter it is known that there is a significant variation in the stress along the axial pin direction. The variation is also found in tight fits and interference fits as reported in e.g. Pedersen (2016). The 3D effect on the stress concentration is neglected in the present paper.

For the circular design a number of papers discuss the stress level in a pinned connection found both from experimental studies, see e.g. Frocht \& Hill (1940), and using analytical work, see e.g. Theocaris (1956), and more recently using numerical methods, see e.g. Wang (1994), Strozzi, Baldini \& Nascimbeni (2006), Pedersen (2007) and Strozzi, Baldini, Giacopini, Bertocchi \& Bertocchi (2011). In many of these publications charts of stress concentration can be found also for different angles of attack for the load. The design is in all cases circular and no design optimization is performed, focus is on the stress level evaluation.

The present paper aim is to minimize the pinned connection stress concentration whereby an increase in the connection strength is achieved, i.e. we optimize with respect to fatigue due to a dynamic/oscillating load. The stress evaluation is performed using the finite element method (FEM) as the numerical tool. To validate that a design improvement has been achieved a significant number of points need to be taken into consideration, these include:

- The stress concentration factor definition is important. The main point is which area the nominal stress is related to, i.e. the pin, the lug net area or the total lug width.

- The needed finite element mesh refinement used in the non-linear contact analysis.

- Influence from head distance.

- Influence from value of Poisson's ratio.

- Influence from modelling assumption, plane stress or plane strain.

- Influence from friction on the maximum stress concentration.

- Clearance and possible comparison to Hertzian pressure.

Design optimization using shape optimization in relation to reduced stress concentrations can be found in many papers. The special case of the present work is that the shape to be optimized also is the shape where the contact takes place between the two parts. Generally it is such that the shape is optimized if the stress level (the maximum stress) along major parts of the shape is constant. The evaluation of stress is here performed using FEM, and as stated in Ding (1986) it is important that the shape parameterization is not done by the FE nodal positions but by an independent parameterization that controls the nodal positions instead. In this way we will not optimize the FEM errors and at the same time a parameterization with a much lower number of parameters can be used, also leading to a design that can be easily communicated. With the shape fixed, either numerically or analytically, a mesh refinement can more simply be done to verify the stress level. The selected parameterization is here the super elliptical one which previously have been used to minimize stress in machine element related components, see e.g. Pedersen (2016) or Pedersen (2018). Shape optimization of a hole in an infinite plate for external loading is well known and in the literature the reported optimal shape is the elliptical design where the ratio between the lengths of principal axes is equal to the ratio between the 
two loads along the principal axes. To the authors knowledge no optimized design for the pin in the lug joint is reported in the literature.

The paper is organized as follows. In Section 2 the definition of stress concentration factor is discussed, specifically in relation to the subsequent evaluation of optimized designs. Section 3 presents the non-linear numerical modelling and important parameters in the modelling. The importance of the head distance is presented in Section 3.1, i.e. the exterior designs influence on the maximum stress level. Section 3.2 presents the influence from Poisson's ratio on the stress level and also the modelling assumption of either plane stress or plane strain is discussed. The inclusion of friction in the connection is shortly discussed in Section 3.3 before Section 3.4 discuss the importance of including clearance in the modelling, a comparison to Hertzian pressure is also given. The shape optimization is presented in Section 4 before the conclusion in Section 5.

\section{Definition of stress concentration factor}

For a pinned connection or lug joint there is not one stress concentration factor definition that is valid for all cases, as also discussed in Pilkey (1997). In Figure 1 a lug joint is shown with dimension, the perpendicular thickness is assumed to be, $T$.

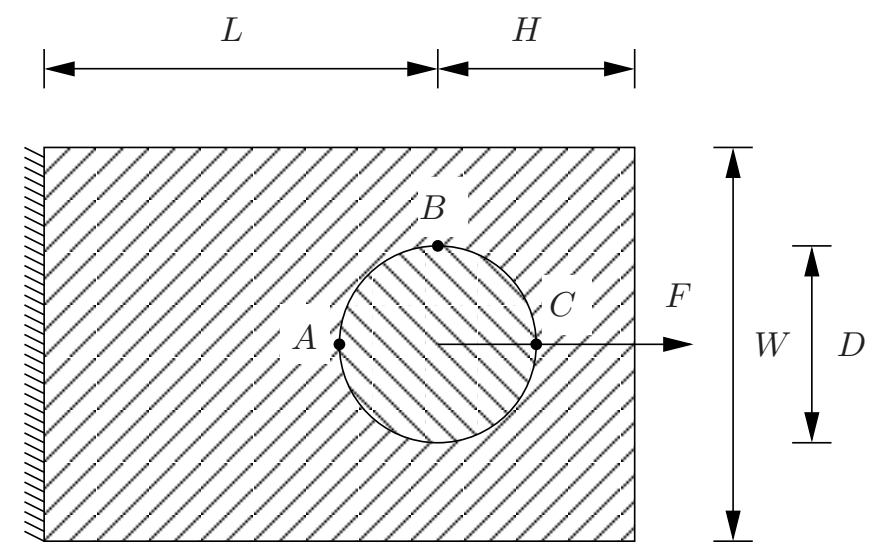

Figure 1: Dimension definition of lug with circular pin, indicating tree points $A, B$ and $C$ along the hole boundary.

Traditionally the theoretical stress concentration factor (index $t$ for theoretical) is defined as

$$
K_{t}=\frac{\sigma_{\max }}{\sigma_{\text {nom }}}
$$

where $\sigma_{\max }$ is the maximum normal stress, i.e. the numerical largest principal stress. The nominal stress definition, $\sigma_{\text {nom }}$, is in principal not important for evaluating the maximum stress from a given stress concentration factor as long as the definition is known. A stress concentration factor should however also preferably indicate how large the variation in the stress is. For the stress concentration value to give a direct physical interpretation it is important that the nominal stress definition do not make the stress concentration factor unreasonably low or high. A common choice for the nominal stress in a pinned connection is to relate it to the net area (of the lug)

$$
\sigma_{\text {nom }}^{n}=\frac{F}{(W-D) T}
$$


i.e. relating the stress concentration to the lug strength with a pin hole. For small values of $W / D$ the stress concentration will be low, although the variation in the stress level is high. For this reason one could also choose to use the bearing area instead

$$
\sigma_{\text {nom }}^{b}=\frac{F}{D \cdot T}
$$

i.e. relating the stress concentration indirectly to the pin strength. For large values of $W / D$ the stress concentration will be low, although the maximum stress is not. Both relating the stress to the net area or to the pin area fails in giving a stress concentration measure that is reasonable for the whole variation range of $W / D$. Relating instead the stress concentration to the strength of the lug without a hole (for the pin) the nominal stress can be defined as

$$
\sigma_{\text {nom }}^{l}=\frac{F}{W \cdot T}
$$

The latter choice is made in the present paper, as also done in Theocaris (1956). The reason being that this gives a direct measure for what the increase in stress is relative to a connection (with width, $W$, and thickness,$T$ ) without a pin. This stress concentration factor is also best suited for evaluating optimized designs, i.e. what is the optimal ratio of $W / D$. It is clear that there is a direct relation between the three nominal stress definitions and the stress concentrations

$$
\sigma_{\text {nom }}^{l}=\frac{1}{\frac{1}{\sigma_{\text {nom }}^{n}}+\frac{1}{\sigma_{\text {nom }}^{b}}}
$$

Leading to

$$
K_{t}^{l}=K_{t}^{n}+K_{t}^{b}
$$

where $K_{t}^{l}=\sigma_{\max } / \sigma_{\text {nom }}^{l}, K_{t}^{n}=\sigma_{\max } / \sigma_{\text {nom }}^{n}$ and $K_{t}^{b}=\sigma_{\max } / \sigma_{\text {nom }}^{b}$.

In Figure 2 the experimental results from Frocht \& Hill (1940) and the analytical results from Theocaris (1956) are shown as they are presented in Pilkey (1997). The analytical results are based on an assumption of a strip, i.e. 2D, and infinite size of $H$ and $L$, see Figure 1 . The experimental results are performed with a size of $H$ and $L$ sufficiently large so that they do not influence the stress level. In Figure 2 the stress concentration as defined in (6) is also shown. From this line it can be seen that there is an optimum with lowest stress concentration for given width, $D$, around $W / D=2.5$ as also described in Theocaris (1956).

If the stress is multi-axial a different stress concentration factor definition might be more appropriate, i.e. in a Hertzian contact problem it is known that the maximum principal stress is at the contact surface but the largest equivalent stress measured by the von Mises stress, $\sigma^{v M}$ is below the surface. So for multi-axial stress a stress concentration defined relative to the von Mises stress might be a better choice.

$$
K_{v M}=\frac{\sigma_{\max }^{v M}}{\sigma_{\text {nom }}^{v M}}
$$




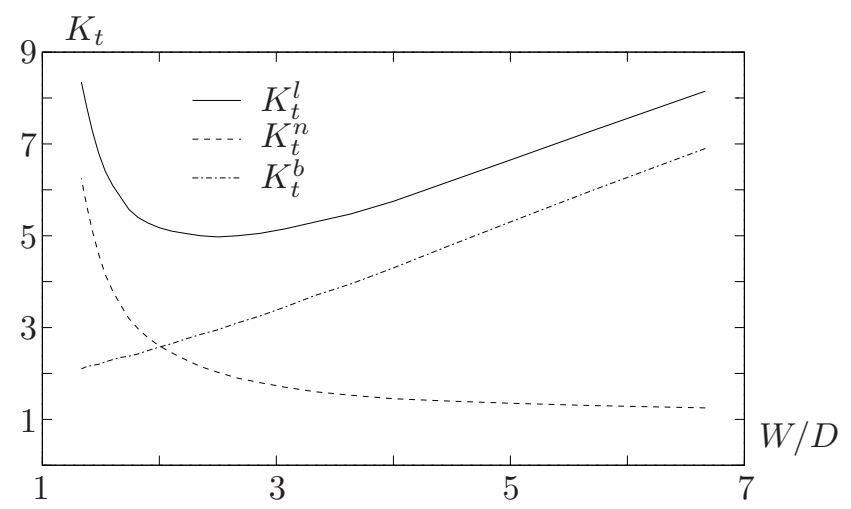

Figure 2: The experimental stress concentration for lug with circular pin from Frocht \& Hill (1940) and the analytical results from Theocaris (1956) as they are shown in Pilkey (1997) ( $K_{t}^{n}$ and $\left.K_{t}^{b}\right)$ together with the curve for $K_{t}^{l}$ derived from the two other curves and Eq. (6).

For the specific cases used in the present paper we will choose to use a stress concentration defined as

$$
K_{v M}^{l}=\frac{\sigma_{\max }^{v M}}{\sigma_{\mathrm{nom}}^{l}}
$$

i.e. the nominal stress is given relative to the lug area as if there were no pin and in this case this will also correspond to the von Mises stress since it is the only stress component.

\section{Numerical modelling and important parameters}

The stress evaluation in pinned connections is in the present paper performed numerically using the finite element method (FEM). The numerical tool used is the COMSOL program COMSOL AB (1986 -). Due to symmetry only half the lug/pin connection is modelled. A high number of elements in the contact are used in order for the stress to converge, which have been checked by mesh refinements. The elements used are triangles with a linear or quadratic displacement assumption. The modelling is done by an assumption of plane stress or strain as discussed later. An example of the used mesh is given in Figure 3.

Symmetry conditions are put on the lowest boundary and the pin is fixed in the centre, a prescribed horizontal displacement is applied to the left boundary, see Figure 3. The maximum stress is evaluated at the contacting zone, i.e. not at the displaced left boundary. The size of the contact area between the two parts is determined as part of the non-linear finite element analysis (FEA).

\subsection{Influence from head distance}

It is initially assumed that there is no clearance between pin and lug i.e. a tight fit. The stress concentration factor is not only controlled by the hole and pin design but also from the other geometric quantities as the ones seen in Figure 1. Keeping the ratio $W / D$ constant and assuming $L$ large enough for the stress to be fully developed towards the left boundary in Figure 1 there is still an influence on the maximum stress from the head distance $H$. This influence was also commented on in the experimental work presented in Frocht \& Hill (1940). It is clear that when $H$ approach $D / 2$ there will be a large influence on the connection strength due to the possibility 
a)

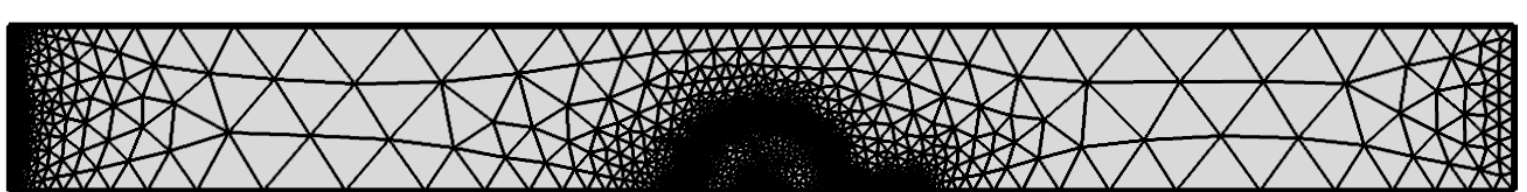

b)

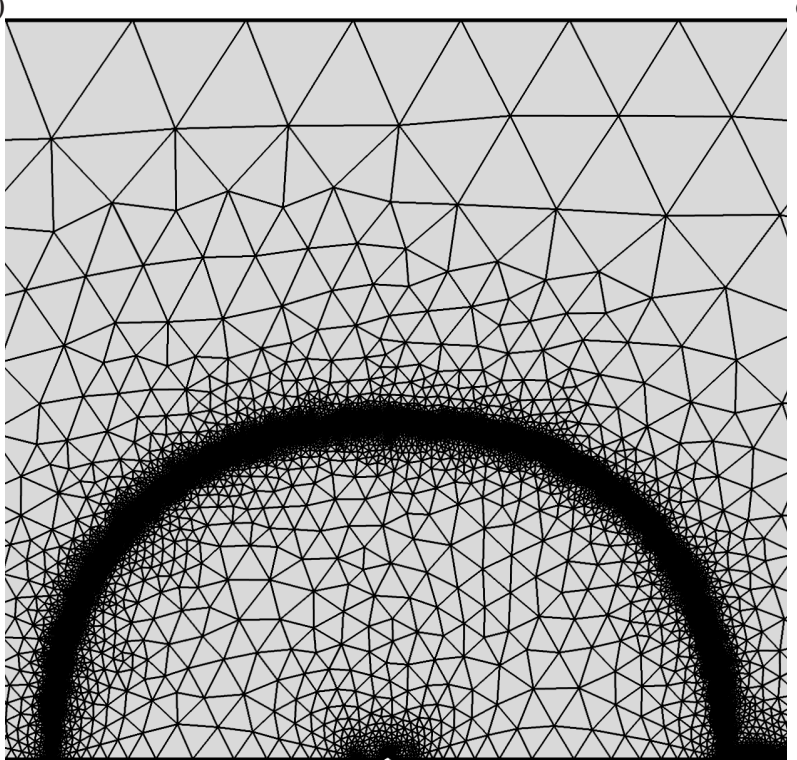

c)

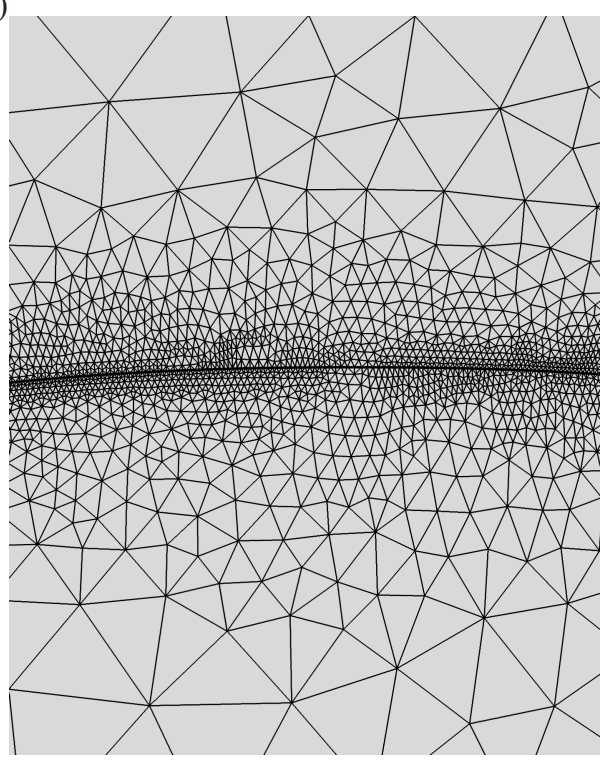

Figure 3: Mesh used for analysis. a) Full mesh, the left boundary is seen to have a fine mesh in order to evaluate the force. The boundary on the bottom is the symmetry line. b) Zoom of pin, the small cut at the centre of the pin is where the constraint is applied. The contact zone is shown to have a fine mesh. c) Zoom of part of contact zone close to point $B$ in Figure 1 .

of pulling out the pin. For large values of $H$ where a fully developed stress field is found in the head (in front of pin) there is no influence on the stress concentration by a further increase in $H$, however the influence from reducing the size of $H$ to the somewhat intuitive choice

$$
H=\frac{W}{2}
$$

is large.

In Figure 4 the stress concentration is shown as a function of the relative head distance for different width to diameter ratios. From the results it is clear that the head distance has a large influence for lower values of $H / D$. For larger values the stress concentration becomes constant as expected and we see that the transition point to constant maximum stress depends on the ratio $W / D$. The lowest stress concentration is found for the value $W / D=2.2$, but for lower value of $H / D$ the optimal value of $W / D$ is changing.

In Figure 5 the stress concentration is shown as a function of width to diameter ratio for the case of head distance being large enough so that it has no influence on the stress level. This curve can be compared to the full line in Figure 2, the overall findings are that we find a lower stress concentration level than that reported in Pilkey (1997). The reason for this can be multiple but primarily properly related to clearance in the case of experimentally found values or the $2 \mathrm{D}$ modelling. The graph is split in three (between point $A$ and $B$, point $B$, point $C$ ) indicating where the maximum stress occurs, see Figure 1. If the maximum stress is between point $A$ and $B$ there is no contact at the point of maximum stress so the von Mises stress is identical to the maximum principal stress when an assumption of plane stress is used. Point $B$ indicates the transition point from contact to no contact also here the von Mises stress is identical to 


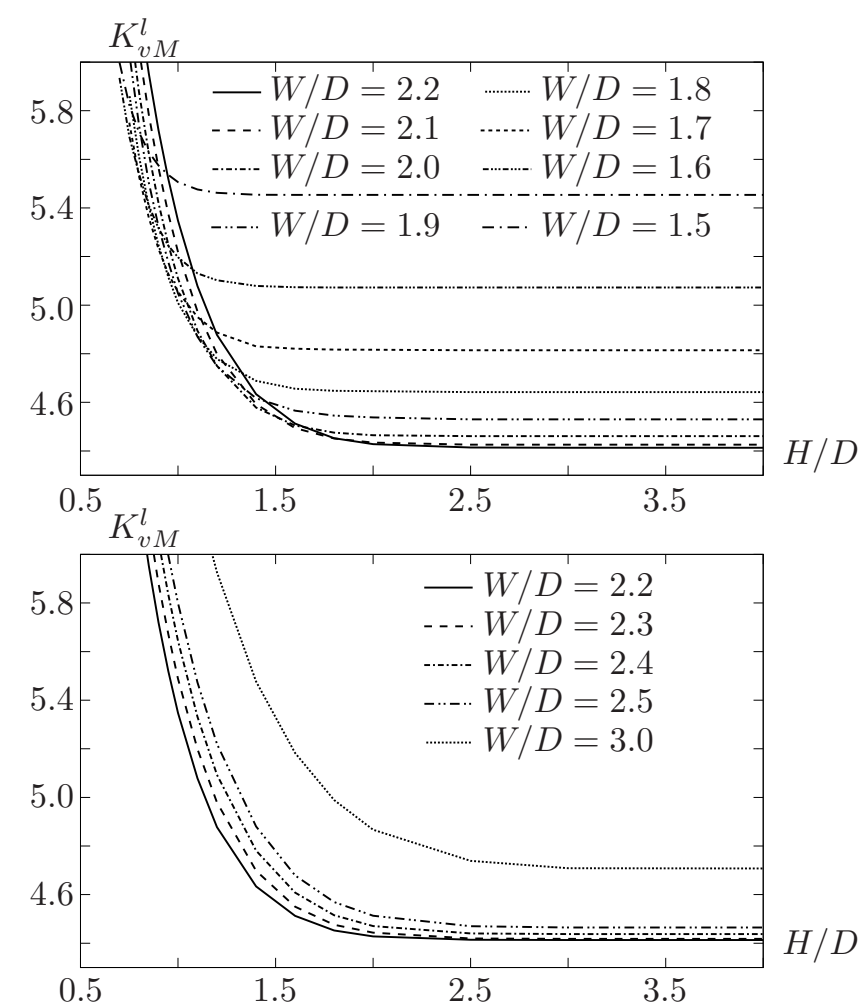

Figure 4: Stress concentration factor as a function of head distance, under plane stress condition (FEA results).

the maximum principal stress, as seen in Figure 5 there is a continuous change between the two areas. At point $C$ the von Mises stress is not identical to the maximum principal stress because of the contact pressure. In the figure we notice the discontinuity in the stress slope at the transition point (around $W / D=3.45$ ).

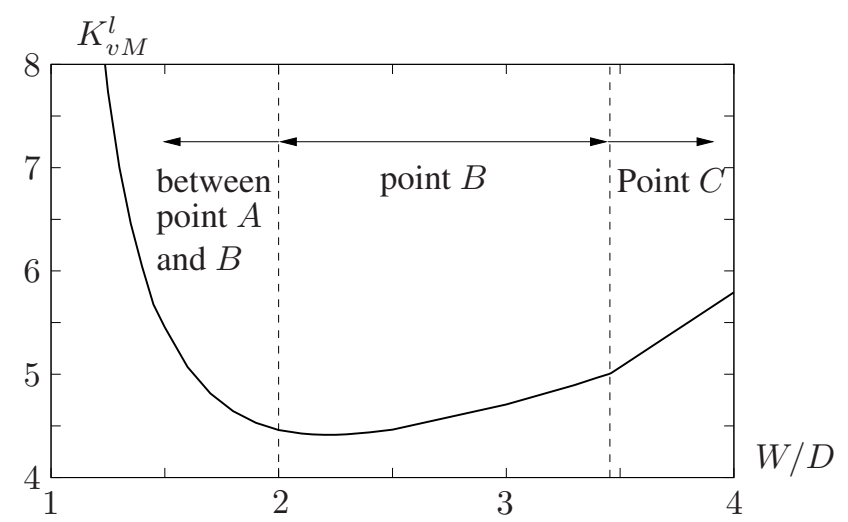

Figure 5: Stress concentration factor as a function of width to diameter ratio, under plane stress condition (FEA results).

\subsection{Influence from Poisson's ratio and plane stress/plane strain assump- tion}

It is well known that Poisson's ratio has an influence on the stress concentration factor value, see e.g. Dally \& Riley (1991) or more recently Pedersen (2018). The previously shown results in this paper have all been found using the Poisson's ration $\nu=0.3$. For the case of $W / D=2.2$ 
and large head distance we show in Figure 6 the stress concentration factor as a function of Poisson's ratio for the case of plane stress and plane strain.

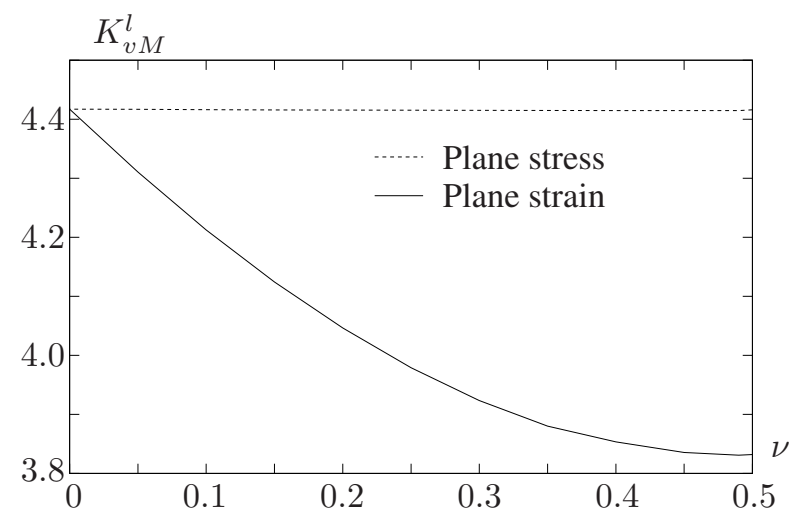

Figure 6: Influence on stress concentration factor from Poisson's ratio and assumption of either plane stress or plane strain $(W / D=2.2)$. (FEA results).

For the specific design with $W / D=2.2$ the maximum stress is at the hole rim at a plane with no contact, i.e., we have a uniaxial stress state for the plane stress assumption and therefore no influence from Poisson's ratio on the stress concentration factor. With the assumption of plane strain Figure 6 shows an influence from Poisson's ratio due to the now double axial stress state at the point with maximum stress. We also see as expected that the results for plane strain and stress are identical for $\nu=0$. If a different design is selected, $W / D=8.0$, we have that the maximum stress is found at the top of contact (point $C$ ), i.e., here we do not have unidirectional stress, independent of the 2D analysis type selected. Figure 7 shows the results.

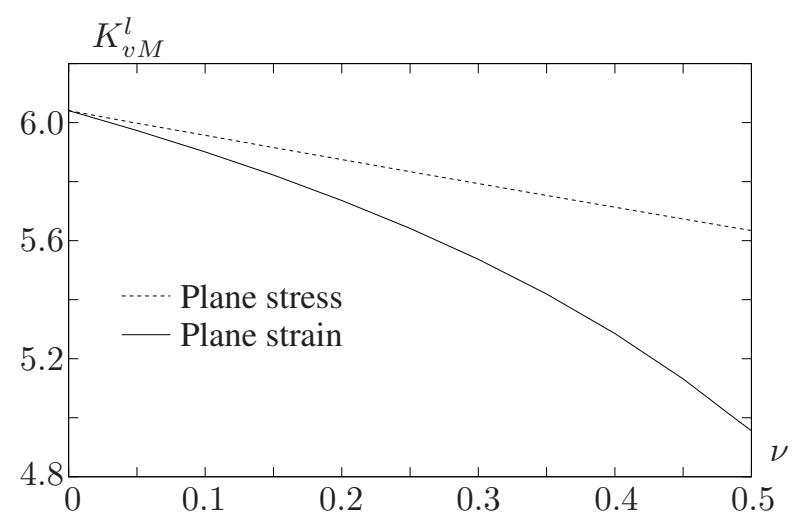

Figure 7: Influence on stress concentration factor from Poisson's ratio and assumption of either plane stress or plane strain $(W / D=8.0)$. (FEA results).

For the case shown in Figure 7 we have with the assumption of plane stress a double axial stress state at the point with maximum stress and as seen the stress concentration factor depends on Poissons ratio to a large extend. For the plane strain case we have a general stress state at the point of maximum stress and as indicated a large influence on the maximum stress from Poisson's ratio. In many cases it is therefore essential that the Poisson's ratio is specified for evaluating the correct stress concentration factor. In the remaining part of the paper we will assume $\nu=0.3$. 


\subsection{Influence from friction on the maximum stress concentration}

The inclusion of Coulomb friction in the contact modelling has shown, for the examples of the present paper, to have no influence on the result. The examples include both assumptions of either plane stress or plane strain and design cases where the point of maximum stress is a point with uni-axial, double axial or a general stress state. The inclusion of friction in the contact modelling is therefore omitted in the remaining examples.

\subsection{Clearance and comparison to Hertzian pressure}

Clearance between pin and lug can have a large influence on the maximum stress in the connection. Discussion on this influence can e.g. be found in Pedersen (2007) and more recent in Strozzi et al. (2011) where also the load direction relative to the lug is discussed.

The selected 2D modelling (with Poisson's ration $\nu=0.3$ ) has a large influence on the maximum stress found. With plane stress assumption, as used in Strozzi et al. (2011), the maximum stress is found on the hole rim in the lug. However, with a plane strain assumption the maximum stress (von Mises) is for low values of the applied force found in front of the load direction but inside the lug, this is in agreement with the traditional Hertzian stress. The stress situation is in the pin/lug connection slightly different than the traditional Hertzian contact because the stress in the lug has to go around the hole into the support.

The Hertzian stress, see e.g. Gladwell (1980) or Norton (2006), is based on an assumption of plane strain, i.e. an infinite half space. For parallel cylindrical contact, as for the pin/lug connection, the half-width of contact is

$$
L_{c}=\sqrt{\frac{2}{\pi}\left(m_{1}+m_{2}\right) \phi \frac{F}{T}}
$$

where $F$ is the contact force (load on pin), $m_{1}$ and $m_{2}$ are material constants which for the case of same material for lug and pin are given by

$$
m_{1}=m_{2}=\frac{1-\nu^{2}}{E}
$$

with $E$ being the modulus of elasticity. $\phi$ is a geometric constant expressing the radius difference for the two bodies. The expression for $\phi$ can also be found in the references Gladwell (1980) and Norton (2006). For the pinned connection we have a concave contact, and assuming the hole diameter in the lug to be given by $D$ and the pin diameter by $D-\delta$ where $\delta$ is the diametral clearance the constant $\phi$ is defined as

$$
\phi=\frac{D(D-\delta)}{\delta} \approx \frac{D^{2}}{\delta}
$$

where the last approximation holds for a relatively small clearance. $T$ is the thickness (as define previously), i.e. the length of the contact zone in the axial direction of the pin. Perpendicular to this direction the distribution of the pressure is semi-elliptical and with the half-width known the maximum pressure at the centre is given by.

$$
p_{\max }=\frac{2 F / T}{\pi L_{c}}
$$


The point of maximum stress depends on the value of Poisson's ration. With $\nu=0.3$ the maximum von Mises stress is found approximately $0.704 L_{c}$ below the point of maximum pressure and is given by (the principal stresses are given in the appendix).

$$
\sigma_{\max }^{v M} \approx 0.558 p_{\max }
$$

Two primary geometric factors control the stress concentration factor size, firstly the shape locally at the point of maximum stress and secondly the amount of load that is transferred to the point of maximum stress. The second factor is controlled by geometric quantities not at the point of maximum stress. In Strozzi et al. (2011) and references therein, the stress concentration factors for lug/pin connections are given as a function of a defined load factor. In the present paper we use the inverse ratio of the load factor and the diametral clearance instead of the radial clearance. The corresponding load factor is here defined as

$$
\psi=\frac{E \delta}{F / T}
$$

The use of this load factor is under the assumption that the load level at the point of maximum stress is given, i.e. the external dimensions of the lug/pin connection is given, and secondly that the nominal shape at the contact is similar. These two points are in agreement with the two primary geometric factors described before. If we define the stress concentration from Hertzian stress we get

$$
\sigma_{\max }^{v M} \approx 0.558 \frac{1}{\sqrt{\pi\left(1-\nu^{2}\right) D(D-\delta)}} \sqrt{E \delta \cdot F / T}
$$

and with the definition (6) we have

$$
K_{v M, \mathrm{Hertz}}^{l} \approx \frac{0.558}{\sqrt{\pi\left(1-\nu^{2}\right)}} \frac{W}{D} \sqrt{\frac{E \delta}{F / T}}
$$

We see that the stress concentration derived from Hertzian stress is approximately linearly dependent of the square root of the load factor. In Figure 8 we show for given material data (steel), $E=210 \mathrm{GPa}$ and $\nu=0.3$, the stress concentration factor as a function of the load factor for two different ratios of $W / D$ and for the plane strain and plane stress case.

The results presented in Figure 8 are found for the specific clearance $\delta=0.001 D$ and the change in load factor is controlled by the forced displacement size (indirectly the load size). The results presented in Figure 8 for the plane stress case and $W / D=2.2$ are in agreement with the results found in Strozzi et al. (2011), i.e. we have (on the log-scale) a linear relationship for high load factor values between stress concentration factor and load factor. For this part of the curve the point of maximum stress is at point $C$. At the kink in the curve the point off maximum stress moves to point $B$ where there is no contact. For low load factor values the stress concentration factor converges almost towards the result for no clearance. For the plane strain case we see the reduction in the stress as also found previously, and we see for low load factors that the stress concentration factor converges towards the no clearance solution. We notice that for the case with $W / D=8$ the minimum stress concentration factor is not found for zero load factor. 


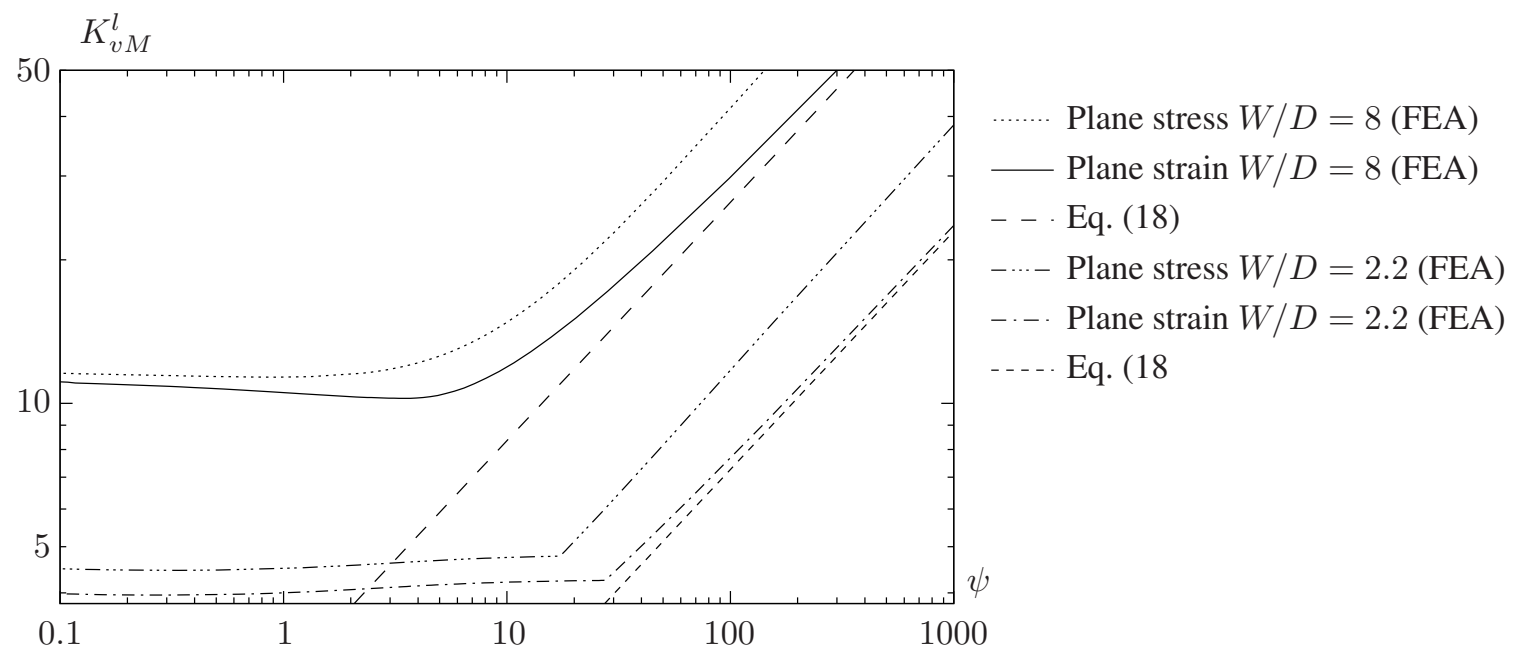

Figure 8: Stress concentration factor as a function of load factor $\psi$ (log-scale).

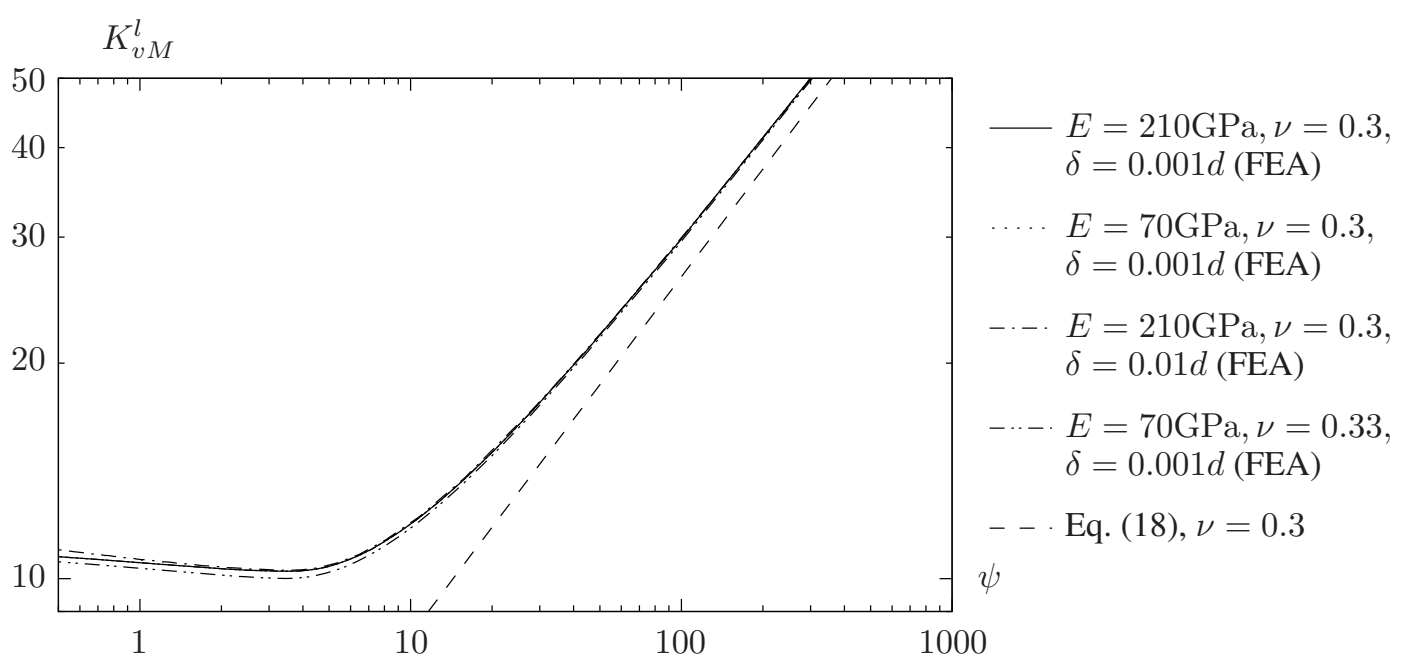

Figure 9: Stress concentration factor as a function of load factor $\psi$ (log-scale) for different material and geometric parameters but with $W / D=8$.

To verify the use of the load factor $\psi$ we show in Figure 9 different curves for the plane strain case.

Overall the numerical results of the present paper support the use of the load factor, $\psi$, but from Figure 9 some caution is suggested. The caution is primarily related to the low load factor $(\psi<10)$ values. The value of Modulus of elasticity has no influence on the stress concentration factor, the dotted line in Figure 9 lies on top of the full line. The clearance size has an influence on the stress concentration factor and for larger relative clearance the result will not converge towards the no clearance result for $\psi=0$. The influence on the result is also noted for even smaller changes in Poisson's ratio.

The relevance of the stress concentration curves in Figures 8 and 9 should also be seen relative to the overall stress level when we design in relation to fatigue failure. If we assume normal production tolerances then we can expect the clearance to be less than $\delta=0.002 D$ for $D>10 \mathrm{~mm}$. For this size of clearance the stress concentration of interest is before the kink in Figure 8, i.e. it is the low stress concentration value that is of interest and here the stress concentration is more or less constant. The modelling and the stress concentration evaluation can therefore be made without including clearance. This observation is important for the optimization performed in the next section. 


\section{Shape optimization of pin/lug connection}

For the plane stress case the most favourable design with tight fit and circular design is $W / D=$ 2.2 , for this design the variation in the von Mises stress in the lug along the hole boundary is shown in Figure 10 (for half the hole due to symmetry). The stress concentration factor is shown to be 4.41 . It is clearly seen that the design cannot be optimal because of the large variation in the stress and also that the maximum stress is only found in one specific point.

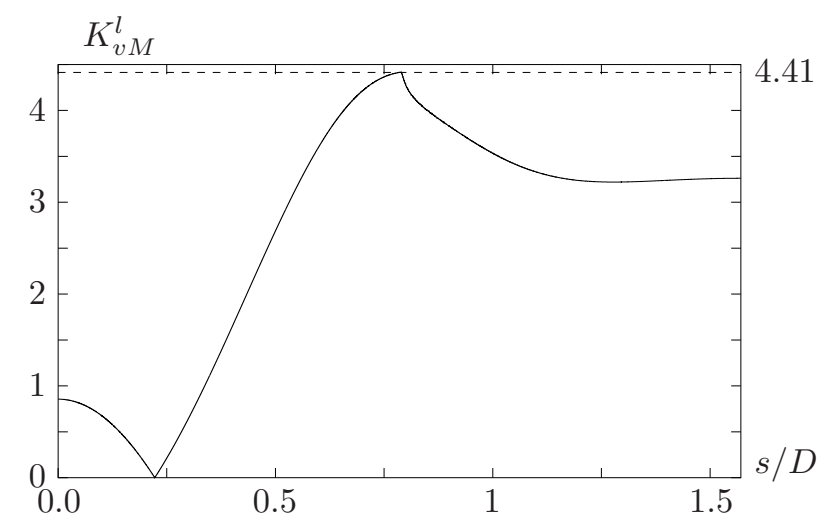

Figure 10: Stress variation along fillet in shaft for the plane stress case. Here $s$ is the arc length along the fillet measured from point $A$ to point $C$ in Figure 1. (FEA results).

In previous papers, see e.g. Pedersen \& Pedersen (2008), Pedersen (2011), Pedersen (2013) and Pedersen (2015) where the maximum stress is minimized for different machine elements, it has been shown that the super elliptical shape and variations of this is an excellent choice for designing a shape with minimum stress concentration.

The super ellipse with principal axes $a$ and $b$ and super elliptical power $\eta$ is in parametric form (for one quarter hole due to symmetry) given by

$$
\begin{array}{ll}
X=a \cos (t)^{(2 / \eta)}, & t \in\left[0: \frac{\pi}{2}\right] \\
Y=b \sin (t)^{(2 / \eta)}, & t \in\left[0: \frac{\pi}{2}\right]
\end{array}
$$

assuming that the $X-Y$ coordinate system origin is at the pin centre and the $X$ coordinate direction is aligned with the force in Figure 1. Due to only three design parameters, $a, b$ and $\eta$, we can perform simple parameter studies, i.e. without sensitivity analysis to find an optimized design. The only constraint on the design parameters is $b<W / 2$ but this constraint will not be active.

For the plane stress case the optimization leads to the design:

$$
a / D=1.16 \quad b / D=1.00 \quad \eta=2.27 \quad W / D=2.2
$$

The variation in the von Mises stress in the lug along the hole boundary is shown in Figure 11. The maximum stress concentration factor is here $K_{v M}^{l}=3.59$ as indicated on the figure. The stress concentration, see Figure 10, is reduced by a factor of $18.6 \%$.

The stress distribution in the lug close to the pin is shown in Figure 12.

For the plane strain case the most favourable design with tight fit and circular design is a slightly larger hole $W / D=2.178$ that has a stress concentration factor of $K_{v M}^{l}=3.91$. If we 


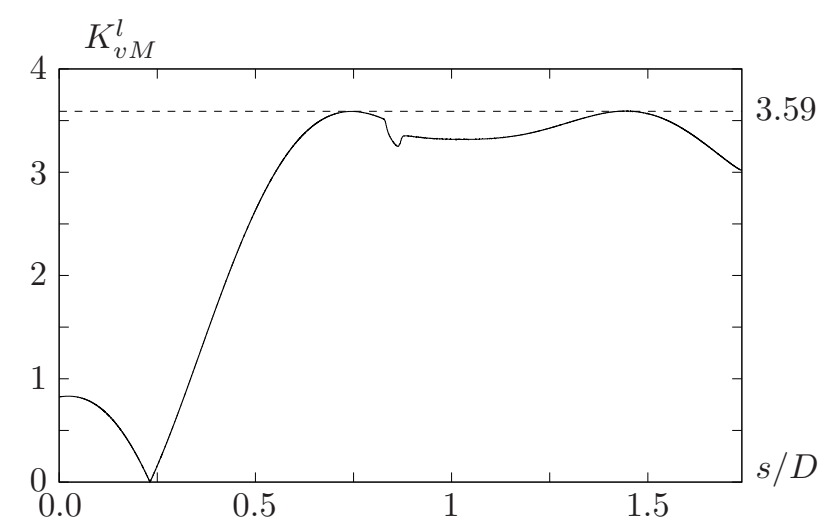

Figure 11: Stress variation along fillet in shaft for the optimized design. Here $s$ is the arc length along the fillet measured along the same direction as for Figure 10. (FEA results).

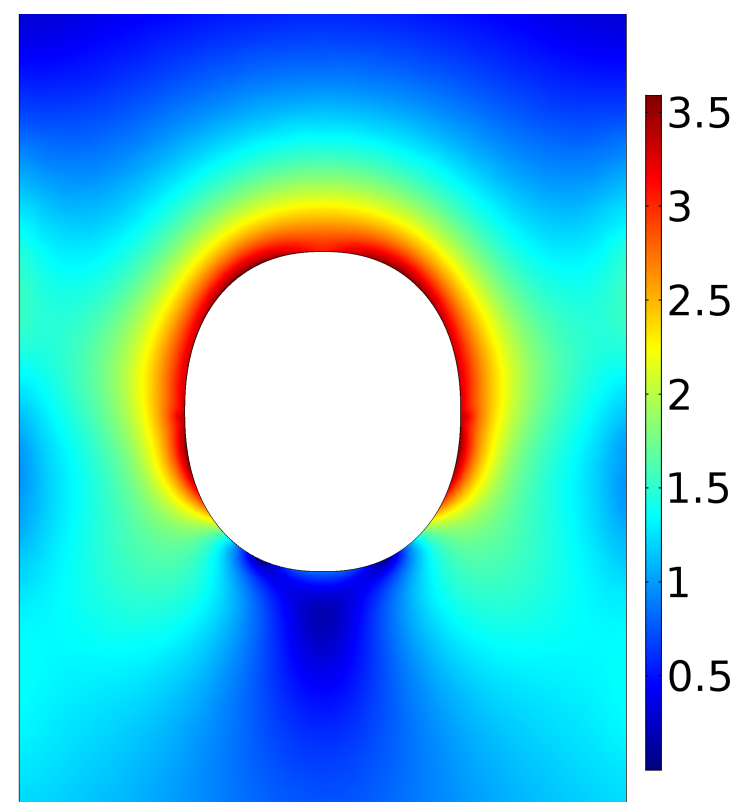

Figure 12: Illustration of stress distribution for optimized shape, scaled with the nominal stress.

specify the width as $W / D=2.2$ as for the previous design case the optimization leads to the optimized design parameters

$$
a / D=1.16 \quad b / D=1.05 \quad \eta=2.27 \quad W / D=2.2
$$

The variation in the von Mises stress in the lug along the hole boundary is shown in Figure 13. The maximum stress concentration factor is here $K_{v M}^{l}=3.36$ as indicated on the figure. The stress concentration is reduced by a factor of $14.1 \%$, i.e. slightly less than for the plane stress case.

It is noticed that the change in the optimized design parameters is very small; only the value of $b$ has been changed slightly.

The optimization has been performed assuming a tight fit and perfect force alignment as seen in Figure 1. For the practical use it might be of interest to know the sensitivity for smaller variation in the load direction. In Figure 14 the stress concentration factor is shown for the circular and optimized design (plane stress case) for a rotation of up to $10^{\circ}$.

As expected the maximum stress for both the optimized design and the circular design are 


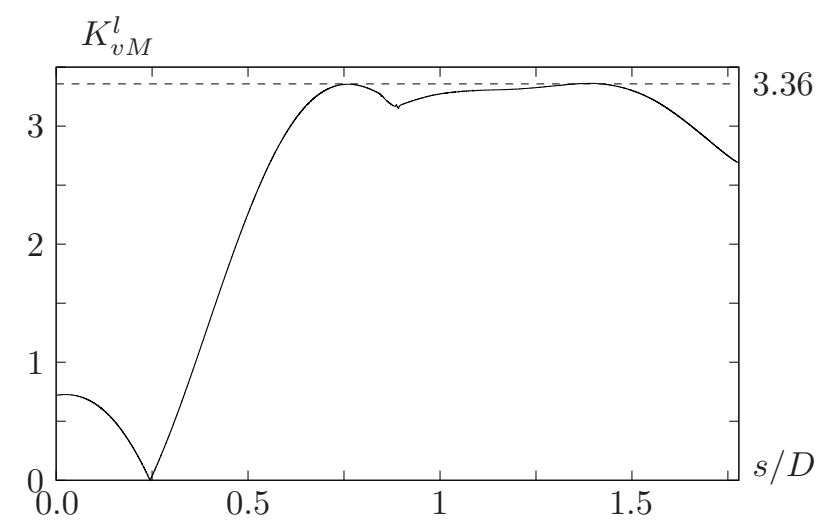

Figure 13: Stress variation along fillet in shaft. Here $s$ is the arc length along the fillet measured along the same direction as for Figure 10. (FEA results).

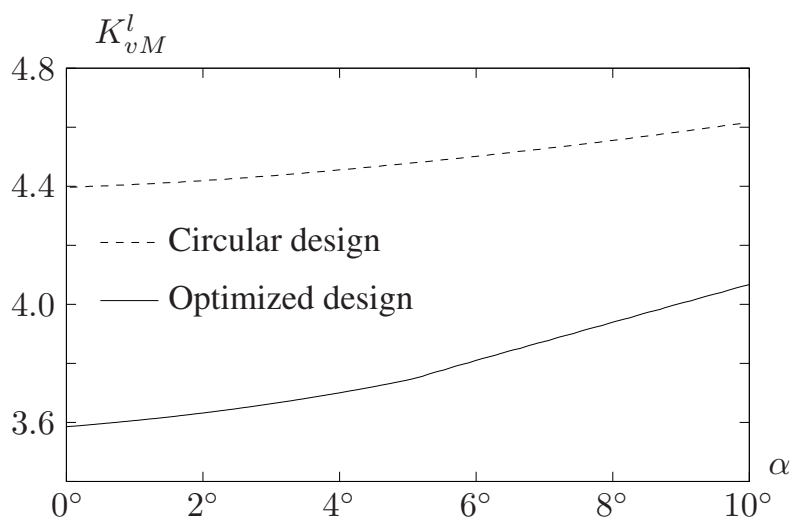

Figure 14: Stress concentration factor in the lug hole (plane stress) as a function of the angle of attack by the external load. (FEA results).

sensitive to the load direction but not to a larger extent for the optimized design that would favour the conventional circular design.

No experimental verification is performed in the present paper of the optimized design. However since the same finite element modelling is used for both the circular and the optimized design we can assume that the relative change or improvement would also be found by physical experiments.

\section{Conclusion}

In the present paper the possibility for shape optimization of the pin/lug connection is examined. The stress evaluation is performed using contact analysis in a non-linear FEA. The stress concentration is shown to be highly sensitive to the size of clearance between pin and hole, but at the same time it is also shown that for practical values of clearance and loads the clearance can be neglected and a tight fit assumption is valid. The value of Poisson's ratio is shown to have (in many cases) a large impact on the stress concentration size, so caution has to be used when applying standard chart for stress concentration where different value of Poisson's ratio might have been used. We show that the head distance is very important for the maximum stress level which is in agreement with the point that two primary geometric factors control the stress concentration factor size: the shape locally at the point of maximum stress and the amount of 
load that is transferred to the point of maximum stress. The head distance contributes to the second point. For the tight fitted pin it is found that the inclusion of friction in the contact modelling can be avoided, the assumption of either plane stress or plane strain has however a large influence on the maximum stress level found.

Using the simple analytical super elliptical shape we show that a $18.6 \%$ reduction of the maximum stress is possible and this reduction is relative to the most preferable circular design. The maximum stress value is sensitive to the load direction for the optimized design but not to a greater extent than the conventional design.

\section{Acknowledgment}

For discussions and suggestions I wish to thank Prof. Peder Klit and Prof. Pauli Pedersen.

\section{A Hertzian stresses for cylinders in contact}

The principal stresses for cylindrical contact (with aligned axis) are according to Hertzian theory, as it may be found in Johnson (1987), given by

$$
\begin{aligned}
& \sigma_{x}=-p_{\max }\left(\frac{2 \bar{z}^{2}+1}{\sqrt{\bar{z}^{2}+1}}-2 \bar{z}\right) \\
& \sigma_{z}=-p_{\max } \frac{1}{\sqrt{\bar{z}^{2}+1}} \\
& \sigma_{y}=\nu\left(\sigma_{x}+\sigma_{z}\right)
\end{aligned}
$$

where $\bar{z}=z / L_{c}$ is the non-dimensional depth below the centre of contact. The principal stress $\sigma_{y}$ follows from the assumption of plane strain. The point of maximum von Mises stress is therefore a function of Poisson's ratio.

\section{References}

COMSOL AB (1986 -), Stockholm, Sweden, www.comsol.com.

Dally, J. \& Riley, W. (1991), Experimental stress analysis, McGraw-Hill,.

Ding, Y. (1986), 'Shape optimization of structures: A literature survey', Computers and Structures 24(6), 985-1004.

DS/EN ISO 2338 (1998), 'Parallel pins, of unhardened steel and austenitic stainless steel'.

Frocht, M. \& Hill, H. (1940), 'Stress-concentration factors around central circular hole in plate loaded through pin in hole', American Society of Mechanical Engineers - Transactions Journal of the Applied Mechanics 7(1), A-5-A-9.

Gladwell, G. M. L. (1980), Contact problems in the classical theory of elasticity, Sijthoff \& Noordhoff.

Johnson, K. L. (1987), Contact mechanics, Cambridge University Press,.

Norton, R. L. (2006), Machine design: An integrated Approach, third edition, Pearson education Inc., Upper Saddle River, N.J. 07458. 984 pages. 
Pedersen, N. L. (2011), 'Optimization of straight-sided spline design', Archive of Applied Mechanics 81(10), 1393-1407.

Pedersen, N. L. (2013), 'Overall bolt stress optimization', Journal of Strain Analysis for Engineering Design 48(3), 155-165.

Pedersen, N. L. (2015), 'Minimizing tooth bending stress in spur gears with simplified shapes of fillet and tool shape determination', Engineering optimization 47(6), 805-824.

Pedersen, N. L. (2016), 'On optimization of interference fit assembly', Structural and Multidisciplinary Optimization 54(2), 349-359.

Pedersen, N. L. (2018), 'Aspects of stress in optimal shaft shoulder fillet', Journal of Strain Analysis for Engineering Design 53(5), 285-294.

Pedersen, N. L. \& Pedersen, P. (2008), 'Design of notches and grooves by means of elliptical shapes', Journal of Strain Analysis for Engineering Design 43(1), 1-14.

Pedersen, P. (2007), 'On the influence of clearance in orthotropic disc-pin contacts', Composite Structures 79(4), 554-561.

Pilkey, W. D. (1997), Peterson's Stress Concentration Factors, 2nd edn, John Wiley and Sons, New York, USA. 508 p.

Strozzi, A., Baldini, A. \& Nascimbeni, M. (2006), 'Maximum equivalent stress in a pinloaded lug subject to inclined loading', Journal of Strain Analysis for Engineering Design 41(4), 297-309.

Strozzi, A., Baldini, A., Giacopini, M., Bertocchi, E. \& Bertocchi, L. (2011), 'Maximum equivalent stress in a pin-loaded lug in the presence of initial clearance', Journal of Strain Analysis for Engineering Design 46(8), 760-771.

Theocaris, P. (1956), 'Stress distribution in strip loaded in tension by means of central pin', American Society of Mechanical Engineers - Transactions - Journal of Applied Mechanics 23(1), 85-90.

Wang, G. S. (1994), 'Stress analysis for a lug under various conditions', Journal of Strain Analysis for Engineering Design 29(1), 7-16. 